\title{
Weiterbildungsbefugnis
}

\section{Versorgungsauftrag des Krankenhauses ist entscheidend}

Nach einem Urteil des Verwaltungsgerichts (VG) München vom 11.03.2014 (Az.: M 16 K 13.1440) kann einem Klinikarzt eine Weiterbildungsbefugnis über den im Krankenhausplan ausgewiesenen Versorgungsauftrag hinaus nicht erteilt werden.

\section{Der Fall: Chefarzt beantragt maximale Weiterbildungsbefugnis (24 Monate)}

Bei dem Kläger handelt es sich um einen Facharzt für Psychiatrie und Psychotherapie. Ab dem 01.01.2010 war er als neuer ärztlicher Direktor und Chefarzt an einer Medizinisch-Psychosomatischen Klinik tätig. Im Vorfeld beantragte er bei der Beklagten die Weiterbildungsbefugnis zum Facharzt für Psychiatrie und Psychotherapie mit Beginn zum 01.01.2010 nach der Weiterbildungsordnung 2004. Mit Bescheid vom Februar 2010 erteilte die Beklagte dem Kläger zunächst eine Weiterbildungsbefugnis im Gebiet Psychiatrie und Psychotherapie bis zur Höchstdauer von 12 Monaten. In der Begründung wurde ausgeführt, der Kammervorstand erteile generell bei Neugründung oder Übernahme einer Abteilung oder Klinik bis zur Vorlage einer unter eigener Leitung erbrachten 12-monatigen Leistungsstatistik nur diesen „Starteffekt“. Im April 2011 beantragte der Kläger sodann erneut eine 24-monatige Ermächtigung für die Weiterbildung in dem Gebiet Psychiatrie und Psychotherapie nach der WBO 2004 und legte unter anderem eine Diagnosestatistik für 2010 vor.

\section{Nur eingeschränkte Weiter- bildungsbefugnis für 6 Monate} Die Beklagte erteilte dem Chefarzt daraufhin mit Bescheid vom April 2012 eine Weiterbildungsbefugnis für 6 Monate mit der Nebenbestimmung, dass die Weiterbildungszeit für Assistenten, die eine Weiterbildung zum Facharzt für „psychoso-
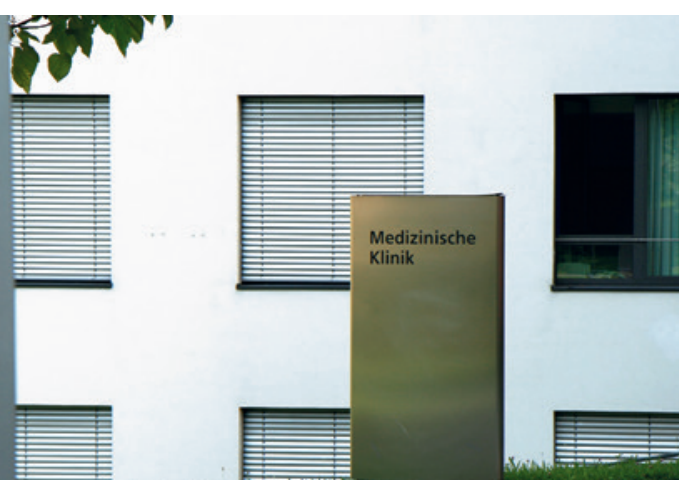

matische Medizin und Psychotherapie“ anstreben, nicht anrechenbar sei. Als Begründung wurde unter anderem ausgeführt, dass die Klinik nicht über eine eigene Abteilung für Psychiatrie und Psychotherapie bzw. zumindest eine fakultativ geschlossene Station zur psychiatrischen Intensivbetreuung bzw. ausschließlich für Patienten mit psychiatrischen Krankheitsbildern ausgewiesene Betten vorhalte. Dies werde auch anhand des Krankenhausplans Bayern bestätigt, wonach für die Klinik keine psychiatrischen Betten ausgewiesen seien. Weiterhin werde an der Klinik keine Poliklinik vorgehalten. Anhand der vorliegenden Unterlagen und einer Begehung hätten sich hinsichtlich der Einrichtung keine Gesichtspunkte ergeben, die eine psychiatrische Schwerpunktbildung erkennen ließen, sodass keine ausreichenden Gegebenheiten vorlägen, um eine Weiterbildung im Fachgebiet der Psychiatrie über einen Zeitraum von 24 Monaten bzw. 12 Monaten sicherzustellen. Der Widerspruch des Klägers gegen diesen Bescheid wurde im März 2013 zurückgewiesen. In der Begründung wurde unter anderem ausgeführt, dass im Ausgangsbescheid nicht angezweifelt worden sei, dass der Kläger zur Weiterbildung fachlich und persönlich geeignet sei. Die Beklagte richte sich bei ihrer Entscheidung nach Beurteilungsrastern, die aufgestellt würden, um im Rahmen einer Qualitätssicherung sämtliche Weiterbildungsinhalte abzudecken und eine Gleichbehandlung aller Antragsteller zu gewährleisten. Klage. In der Klage wurde unter anderem vorgetragen, es handele sich bei dem Beurteilungsraster um rein interne Richtlinien oder Entscheidungskriterien. Nach Auffassung des Klägers könnten die Weiterbildungsinhalte der WBO in der Klinik des Klägers in der Weiterbildungszeit von 24 Monaten in ausreichendem Maße und in einem der 24-monatigen Weiterbildungszeit adäquaten Umfang vermittelt werden.
Gegen diesen Bescheid erhob der Kläger

\section{Klage abgelehnt}

Das VG München lehnte die Klage als unbegründet ab. Dem Kläger sei mit Bescheid vom April 2012 eine Weiterbildungsbefugnis für 6 Monate mit einer Nebenbestimmung erteilt worden. Ein Anspruch auf Erteilung einer weitergehenden Weiterbildungsbefugnis ohne diese Nebenbestimmung stehe ihm nicht zu.

\section{Kläger ist fachlich und persönlich geeignet}

Unstreitig erfülle der Kläger zwar die fachliche und persönliche Eignung, für die Erteilung seien aber weitere Kriterien maßgebend, welche sich auf die betreffende Weiterbildungsstätte beziehen, die vom Kläger bzw. der Weiterbildungsstätte nicht erfüllt werden. Bei diesen Kriterien handele es sich im Einzelnen um den Versorgungsauftrag (Anzahl sowie Erkrankungs- und Verletzungsarten der Patienten), die Leistungsstatistik (Art und Anzahl der ärztlichen Leistungen) sowie die personelle und materielle Ausstattung der Weiterbildungsstätte.

\section{Klinik erfüllt Voraussetzungen nicht}

Die vom Kläger geleitete Klinik erfülle nicht alle diese Kriterien. Gemäß der Festlegung zur Weiterbildungszeit für das Gebiet „Psychiatrie und Psychotherapie“ ist gemäß der Weiterbildungsordnung 2004 eine 24-monatige Weiterbildung in der stationären psychiatrischen und psychotherapeutischen Patientenversorgung erforderlich. Der Begriff der „psychiatrischen und psychotherapeutischen Patientenversorgung“ sei in Übereinstimmung mit den Fachrichtungen der Krankenhäuser nach dem Krankenhausplan des Freistaats Bayern auszulegen. Der Krankenhausplan enthält unter anderem die Fachrichtungen „Psychiatrie und Psychotherapie“ sowie „Psychosomatische Medizin und Psychotherapie“. Dieselbe Differenzierung liegt auch der WBO 2004 zugrunde. Sie unterscheide zwischen psychiatrischer und psychotherapeutischer Fachrichtung einerseits und dem Gebiet der psychosomatischen Medizin und Psychotherapie andererseits. 


\section{Zuordnung im Krankenhausplan mit entscheidend}

Die vom Kläger geleitete Klinik sei im geltenden Krankenhausplan der Fachrichtung „Psychosomatische Medizin und Psychotherapie“ zugeordnet. Nach dieser auch gegenüber dem Krankenhausträger verbindlich getroffenen Feststellung dient dieses Krankenhaus der bedarfsgerechten Versorgung der Bevölkerung im Hinblick auf diese Fachrichtung, nicht dagegen der psychiatrischen und psychotherapeutischen Patientenversorgung. Folglich habe eine Weiterbildungsstätte im Bereich der stationären psychiatrischen und psychotherapeutischen Patientenversorgung auch über eine psychiatrische Abteilung zu verfügen. Es sei dagegen nicht ausreichend, dass - wie im Fall der vom Kläger geleiteten Klinik Patienten mit psychiatrischen Krankheitsbildern auch unter psychiatrischen Aspekten in Abteilungen behandelt werden, die einer anderen Patientenversorgung dienen. Zudem habe eine solche Weiterbildungsstätte auch ein vollständiges psychiatrisches Curriculum anzubieten, das alle für die psychiatrische Patientenversorgung relevanten Inhalte abdecke.

Soweit der Kläger dem entgegenhielt, dass er nicht das gesamte psychiatrische Curriculum und das entsprechende Leistungsspektrum abdecken müsse, da er ja auch nur eine teilweise Weiterbildungsermächtigung beantragt habe, folgte ihm das VG nicht. Die beantragte 24-monatige Weiterbildungszeit würde die gesamte Weiterbildungszeit in dieser Art der Weiterbildungsstätte betreffen und müsste daher die gesamten Weiterbildungsinhalte umfassen. Zum anderen sehe die WBO 2004 auch nicht vor, dass ein Teilabschnitt einer Weiterbildungszeit angeboten werden könne, wenn nur ein Teil der Weiterbildungsinhalte vermittelt werde. Die Regelung über die Weiterbildungszeiten in der WBO 2004 gehe vielmehr ersichtlich davon aus, dass eine Weiterbildung in der stationären psychiatrischen und psychotherapeutischen Patientenversorgung stets in Einrichtungen erfolge, die das gesamte psychiatrische Curriculum und Leistungsspektrum abdecken können. Diese Anforderungen der WBO 2004 an geeignete Weiterbildungsstätten seien rechtlich auch nicht zu beanstanden, da sie sich im Rahmen der Einschätzungsprärogative der Beklagten bei Ausgestaltung der Voraussetzung für die Anerken- nung von Weiterbildern und Weiterbildungsstätten aufgrund des HeilberufeKammergesetzes bewege.

\section{Notfallaufnahme fehlt}

Das Gericht weist weiter darauf hin, dass aus den Weiterbildungsinhalten der „Krisenintervention“ sowie dem Bezug zur „Anwendung von Rechtsvorschriften bei der Unterbringung psychisch Kranker“ zu schließen sei, dass eine Notfallaufnahme und eine geschlossene Station bei der Weiterbildungsstätte vorhanden sein müssen, welche die Klinik des Klägers nicht aufweise.

\section{Auch Nebenbestimmung rechtmäßig}

Nach Auffassung des Gerichts ist auch die Nebenbestimmung, dass die 6 Monate Weiterbildungszeit für Assistenten, die eine Weiterbildung zum Facharzt für psychosomatische Medizin und Psychotherapie anstreben, nicht anrechenbar sei, rechtmäßig. Die Weiterbildung im Gebiet Psychiatrie und Psychotherapie könnte nicht als Weiterbildungszeit in der Psychiatrie gewertet werden. Die Nebenbestimmung schließe daher aus, dass eine - nach der WBO 2004 unzulässige Anrechnung der 6-monatigen Weiterbildungszeit für das Gebiet psychosomatische Medizin und Psychotherapie erfolgen kann.

\section{Fazit}

Nach Auffassung des VG München ist für die Weiterbildungsbefugnis maßgeblich, mit welchem Versorgungsauftrag das Krankenhaus in den Krankenhausplan aufgenommen wurde. Ein Krankenhaus kann daher nur als Weiterbildungsstätte in der stationären psychiatrischen und psychotherapeutischen Patientenversorgung anerkannt werden, wenn es im Krankenhausplan dieser Fachrichtung zugerechnet wird und die entsprechenden psychiatrischen Weiterbildungsinhalte vollständig abdeckt.

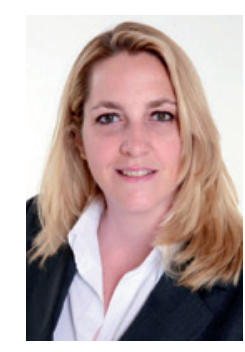

Korrespondenz Dr. iur. Isabel Häser Rechtsanwältin ECOVIS Lüdemann Wildfeuer \& Partner Sonnenstr. 9 80331 München www.ecovis.com 